\title{
Das Grundrecht der brasilianischen Indigenen auf Land: Der Fall Raposa Serra Do Sol
}

\author{
Von Ana Maria D'Ávila Lopes \\ und Martonio Mont'Alverne Barreto Lima, Fortaleza*
}

\section{Einführung}

Nach neuesten Studien umfasst die indigene Bevölkerung Brasiliens ungefähr 460.000 Personen, die auf 225 Gemeinschaften verteilt sind. Diese Daten beziehen sich auf die Menschen, die in Dörfern leben. Es wird geschätzt, dass es zwischen 100.000 und 190.000 geben muss, die außerhalb von diesen leben, auch in städtischen Gebieten ${ }^{1}$. Genau wie auf dem übrigen amerikanischen Kontinent verursachte die europäische Kolonisierung zahllose Missgriffe gegen die Indigenen und ihre Kultur. Diese Situation begann sich erst in den letzten Jahrzehnten zu ändern. Paradigmatischer Moment für diesen Wandel war die Verkündung der Bundesverfassung 1988, mit der die herrschende Assimilierungspolitik aufgegeben wurde, um der Anerkennung verschiedener indigener Rechte Raum zu geben, wie dem Recht auf Bewahrung ihrer Kultur (Artikel 231, caput); dem Recht in der eigenen Sprache und mittels der eigenen Lernprozesse unterrichtet zu werden (Artikel 210, § 2); dem originären Recht auf den Besitz des traditionell von ihnen besetzten Landes und auf den Nutzen der Reichtümer des Bodens sowie der im Gebiet vorhandenen Flüsse und Seen (Artikel 231, caput § 2); und dem direkten Schutz der Staatsanwaltschaft (Artikel 232). Unter diesen Rechten verdient das Grundrecht auf Land ${ }^{2}$ ein eingehendes Studium aufgrund der wesentlichen Rolle, die dieses für die Bewahrung der indigenen Kultur besitzt und da es die wesentlichste ihrer Forderungen darstellt, wie Souza Filho gut hervorhebt: "Das Recht auf Land, verstanden als Raum des Lebens und der Freiheit einer Gruppe von

* Ana Maria D’Ávila Lopes, Master und Doktor in Verfassungsrecht der Universidade Federal de Minas Gerais - UFMG. Professorin des Postgraduiertenprogramms in Rechtswissenschaften der Universidade de Fortaleza -UNIFOR. Forschungsstipendiatin des CNPq.

E-mail:anadavilalopes@yahoo.com.br

Martonio Mont'Alverne Barreto Lima, Master in Rechtswissenschaften der Universidade Federal do Ceará - UFC und Dr. jur (Universität Frankfurt a.M.). Professor Titular des Postgraduiertenprogramms in Rechtswissenschaften der Universidade de Fortaleza - UNIFOR und derzeit Generalstaatsanwalt der Stadt Fortaleza, E-mail: barreto@unifor.br

1 Fundação Nacional do Índio, in: https://www.funai.gov.br/, Zugriff am: 14 set. 2.009.

2 Vgl. Ana Maria D'Ávila Lopes und Karine Mattos, "O direito fundamental dos indígenas à terra: uma investigação científica do Brasil Colônia ao Estado Democrático de Direito", Revista de Informação Legislativa, v. 43 (2006), S. 221-234. 
Menschen, ist eine fundamentale Forderung der indigenen Völker Brasiliens und Lateinamerikas",3.

\section{$1.1 \quad$ Erklärung der Rechtsform}

Die brasilianischen Indigenen gelten nicht als Eigentümer des Landes, das sie traditionell innehaben, sondern lediglich als Besitzer. Das Eigentum des von den Indigenen innegehabten Landes liegt beim Bund (Artikel 20, XI der Verfassung), wobei lediglich das Nutznießungsrecht exklusiv den Indigenen vorbehalten ist (Artikel 231, § 2 der Verfassung). Dieses Modell - Bund als Eigentümer und Indigene als Besitzer - soll die indigene Kultur bewahren, deren Existenz eng mit Grund und Boden verbunden ist. Diese verfassungsmäßige Festlegung vermied eine Verfügungsbefugnis durch die Indigenen, während die Zusicherung des ausschließlichen Nutznießungsrechts an die Indigenen eine Verfügungsbefugnis des Bundes verhinderte. So wurde ein eindeutiges Modell geschaffen, in dem beide Subjekte (Bund und Indigene) sich selbst abgrenzen und jegliches Übermaß vermieden wird, aus dem der Verlust des Landes und folglich das Auslöschen der indigenen Gemeinschaft erwachsen könnte.

\subsection{Rechtsnatur des Rechts der Indigenen auf Land}

Auch wenn das Recht der Indigenen auf Land nicht im Titel II der Bundesverfassung von 1988 steht, der sich auf Grundrechte und Garantien bezieht, handelt es sich um ein Grundrecht nach Artikel 5 § 2. Danach sind Grundrechte a) die, die in der Verfassung zum Ausdruck gebracht sind, also nicht nur die ausdrücklich in Titel II festgelegten, sondern auch die an jeder anderen Stelle des Verfassungstextes, wie das Recht auf Umwelt, das in Titel VIII vorgesehen ist; b) die aus den Verfassungsprinzipien entspringenden, die nicht notwendigerweise nur die sind, die in Titel I aufgenommen sind, sondern an jeder anderen Stelle der Verfassung; c) die aus dem angenommenen politischen Regime, dem demokratischen, abgeleiteten; und d) die, die in internationalen, vom brasilianischen Staat ratifizierten Verträgen über Menschenrechte vorgesehen sind. Im letzten Fall können nach Artikel 5 $\S 3$ die internationalen Verträge immer dann Verfassungsrang haben, wenn sie nach dem gleichen Verfahren angenommen wurden, das für eine Verfassungsänderung verwendet wird (Abstimmung in zwei Lesungen der Abgeordneten und Senatoren mit Dreifünftelmehrheit in beiden Kammern), und so das darstellen, was die Rechtslehre Verfassungsblock nennt ${ }^{4}$.

Carlos Frederico Marés Souza Filho, O renascer dos povos indígenas para o direito, Curitiba, 2005, S. 119. Eigene Übersetzung.

4 Vgl. Ana Maria D'Ávila Lopes, Bloco de constitucionalidade e princípios constitucionais: desafios do Poder Judiciário. Sequência, v.29, (2009), S.43 - 60. 
Flávia Piovesan ${ }^{5}$ bekräftigt bei ihrer Untersuchung von Artikel 5 §, dass sich seine Aufnahme in die Verfassung aus der Anerkennung der ausdehnenden Kraft der Werte der Menschenwürde und der Grundrechte als axiologische Parameter ableitet, die das Verständnis des Verfassungstextes orientieren. Dies ist die begriffliche Grundlage der Grundrechte, die als Rechtsprinzipien von Verfassungsrang definiert werden können, welche die wichtigsten Werte einer jeden Gesellschaft widerspiegeln und dazu dienen, die Menschenwürde direkt zu schützen und so das Handeln des Staates und der Privatpersonen zu legitimieren $^{6}$. Aus der Definition kann gefolgert werden, dass die Grundrechte in dem Maße Normen von höchstem Rang sind mit der Aufgabe, die Würde des ganzen Menschen zu wahren, eine Aufgabe, die Zentrum und Zweck jedes öffentlichen oder privaten Handelns ist. Ihre Funktion, die Menschenwürde zu beschützen, ist das Schlüsselelement, um ein Recht als grundlegend zu charakterisieren. Es ist richtig, dass jedes Recht, jede Rechtsnorm zum Ziel hat, das Wohlbefinden des Menschen zu schützen - oder wenigstens sollte es so sein - aber im Fall der Grundrechte ist dieser Schutz direkt und ohne normative Vermittlung.

Die Bedeutung, das Grundlegende des Rechts der Indigenen auf Land zu erkennen, ist entscheidend, um einen besseren und sicheren Schutz zu garantieren. Verschiedene Verfassungsmechanismen sind zu diesem Zweck bestimmt, wie die Norm, die festlegt, dass jedes Grundrecht und -sicherheit sofortige Anwendung widerfährt (Artikel 5, § 1).

Schließlich kann das Recht der Indigenen auf Land in dem Maße als ein diffuses Grundrecht oder Grundrecht der dritten Generation klassifiziert werden, in dem es einem transindividuellen Recht, unteilbarer Natur, einer unbestimmten Gruppe von Personen entspricht, die durch eine de-facto-Situation vereint sind $^{7}$.

\subsection{Klassifizierung des indigenen Landes}

Die traditionell von Indigenen bewohnten Gebiete sind Güter des Bundes, also öffentliche Güter (Artikel 29, XI der Verfassung). Öffentliche Güter werden nach Artikel 99 des brasilianischen Bürgerlichen Gesetzbuches (Gesetz Nr. 10.406/2002) eingeteilt in a) allgemeinen Nutzens oder gleichsamen Nutzens aller Personen; b) besonderen Nutzens, für einen spezifischen Zweck bestimmt; und, c) Staatsgüter, die das Vermögen von juristischen Personen öffentlichen Rechts als Objekt persönlichen oder realen Rechts jeder einzelnen von diesen Einrichtungen bilden. Aufgrund seiner Eigenschaft als öffentliches Gut besonderen Nutzens ist das indigene Land unveräußerlich (Artikel 100 des brasilianischen Bürgerlichen Gesetzbuchs - Gesetz Nr. 10.406/2002), unverjährbar (Artikel 100 der Bundes-

Flávia Piovensan, Direitos humanos e o direito constitucional internacional, São Paulo, 1995, S. 160.

Ana Maria D'Ávila Lopes, Os direitos fundamentais como limites ao poder de legislar, Porto Alegre, 2001, S. 36-37.

7 Definition des diffusen Rechts, vorgesehen in Abschnitt I des Einzigen Paragraphen von Artikel 81 des Verbraucherschutzgesetzbuchs - Gesetz Nr. 8.078/1990. 
verfassung) und unpfändbar (einziger Paragraph von Artikel 191 der Bundesverfassung). Das traditionell von Indigenen bewohnte Land bildet öffentliches Bundeseigentum und ist folglich unantastbar. Die Verfassung schreibt vor, dass dieses Land unveräußerlich und unverfügbar und die Rechte an ihnen unabdingbar sind. Daher ist das von den Indigenen besetzte Land durch seine Unververäußerlichkeit, Unverfügbarkeit, Unabdingbarkeit und Unverpfändbarkeit gekennzeichnet. Auch ist indigenes Land gegen Enteignungsklagen der öffentlichen Hand immun gemäß Bundesgesetz Nr. 6.001/73, Artikel 38. Das traditionell von den Indigenen besetzte Land bildet eine res extra commercium, da es nicht Gegenstand von Verpachtung, Rechtsakt oder -geschäft sein kann, die die volle Ausübung des direkten Besitzes durch die indigene Gemeinschaft oder die Waldbewohner beschränken ${ }^{8}$. So verfügt auch die Verfassung in Artikel $231 \S 4$, wo festgelegt wird, dass das indigene Land unveräußerlich, unverfügbar und unabdingbar ist.

\subsection{Originale Rechte auf das Land}

Das Indigenat ist eine portugiesisch-brasilianische Institution, die im Alvará Regio vom 1. April 1680 geschaffen wurde und den Indigenen die Eigenschaft als erste und natürliche Herren des Landes von Brasilien zugestand ${ }^{9}$ Das Recht der Indigenen auf Land ist also kein erworbenes Recht, sondern ein originäres oder angeborenes Recht, das heißt, es beginnt und endet mit ihnen ohne die Notwendigkeit einer anderen Voraussetzung als die einfache existentielle Beziehung zum Land. Da es sich um einen Besitz ab origine handelt, also im Bewusstsein der indigenen Gemeinschaften gegenwärtig ist, ist dieser nicht durch das bürgerliche Recht geregelt, sondern gehorcht den anthropologischen Parametern einer jeden indigenen Gemeinschaft.

Andererseits bezieht sich der Ausdruck ,traditionell besitzt“ nicht auf einen uralten Besitz, sondern auf den Besitz des Landes in seiner Eigenschaft als Lebensraum, das heißt, zur Durchführung der produktiven Tätigkeiten und zur Bewahrung der natürlichen Ressourcen bestimmt, die für das Wohlergehen der Gemeinschaft notwendig sind, sowie zur physischen und kulturellen Reproduktion im Einklang mit ihren Gebräuchen, Sitten und Traditionen (Artikel 231, § 1 der Verfassung). Aus diesem Recht wird das Prinzip der Unentfernbarkeit der Indigenen von ihrem Land abgeleitet. Als Ausnahme sind Katastrophenfälle oder Epidemien zugelassen, in denen die Entfernung ad referendum des Nationalkongresses zu erfolgen hat, oder um die nationale Souveränität zu schützen. In diesem Fall ist die vorherige Zustimmung des Nationalkongresses erforderlich, wobei bei allen genannten Voraussetzungen die sofortige Rückkehr der Indigenen in ihr Land nach Ende der Gefahrenlage zu garantieren ist (Artikel 231, § 5).

Luiz Pinto Ferreira, Curso de direito constitucional, São Paulo, 2002, S. 577. Eigene Übersetzung. 


\subsection{Konstitutionelle Nutznießung}

Nach Artikel $231 \S 2$ der Bundesverfassung ist das traditionell von Indigenen besessene Land für ihren dauernden Besitz bestimmt, wobei ihnen die ausschließliche Nutznießung der Ressourcen des Bodens und der auf dem Land existierenden Flüsse und Seen zusteht. Exklusives Nutznießrecht bedeutet, dass die Indigenen alle natürlichen Ressourcen ihres Landes nutzen können, wie die Früchte, Nutzen und Einkünfte, solange diese nicht die Nachhaltigkeit für die Umwelt in einem Maße schädigen, in dem dies das Recht zukünftiger indigener Generationen auf Land beeinträchtigt (Artikel 225 der Verfassung).

Dieses Recht war schon im Indianerstatut (Gesetz Nr. 6.001/1973) in Artikel 24 vorgesehen:

„Artikel 24. Die den Indigenen oder Waldbewohnern garantierte Nutznießung umfasst das Recht auf Besitz, Nutzen und Bezug der auf dem besetzten Land vorhandenen natürlichen Ressourcen und aller Nutzen, sowie auf das Ergebnis der wirtschaftlichen Ausbeutung dieser natürlichen Ressourcen und Nutzen.

§ 1 In die Nutznießung, die sich auf das Zubehör und ihre Hinzufügungen erstreckt, wird der Nutzen der Quellen und der Wasser der Flussabschnitte eingeschlossen, die auf dem besetzten Land erfasst werden.

$\S 2$ Den Indigenen ist die ausschließliche Ausübung von Jagd und Fischerei in dem von ihnen besetzten Gebiet garantiert, wobei die Polizeimaßnahmen auf überzeugende Weise ausgeübt werden müssen, die in Bezug darauf eventuell angewandt werden müssen“.

Andererseits ist hervorzuheben, dass das ausschließliche Nutznießrecht die Indigenen nicht dazu verpflichtet, die Ressourcen direkt auszubeuten, denn es ist ihnen erlaubt, sich mit Dritten zu assoziieren oder sich von ihnen in Projekten beraten zu lassen, die die Ausbeutung ihrer natürlichen Ressourcen zum Ziel haben. Da das indigene Land unveräußerlich ist, können sie sich in keinem Fall in Projekte verwickeln, die den Verlust des Besitzes ihres Landes verursachen oder die Nachhaltigkeit ihrer Ressourcen beeinträchtigen. So ist es sowohl in der Verfassung (Artikel 231, § 6) als auch im Indianerstatut festgelegt (Artikel 18, caput). Andererseits ist bei der Definition des ausschließlichen Nießbrauchs zu unterscheiden: a) das Nutzen der natürlichen Ressourcen, um die internen Bedürfnisse der indigenen Gemeinschaften im Einklang mit ihren Gebräuchen, Sitten und Traditionen zu stillen; b) die Produktion von Überschuss zum Verkauf, auch wenn dies zu ihrer Subsistenz geschieht. Im ersten Fall können die Ressourcen und Güter in dem Maße uneingeschränkt verwendet werden, in dem aufgrund der indigenen Kosmologie selbst keine Gefahr besteht, dass sie irgendeinen Exzess gegen die Natur begehen. Dies geschieht aufgrund des Respekts, den sie für die Natur hegen, denn letzten Endes haben die Indigenen keine anthropozentrische Sicht der Natur, wie sie in der westlichen Kultur präsent ist ${ }^{10}$. Im zweiten Fall hingegen muss die Gewinnung dieser Überschüsse die Umweltschutznormen

10

Vgl. Eduardo Viveiros de Castro, Perspectivismo e multinaturalismo na América indígena, São Paulo, 2002. 
beachten, das heißt, sie ist durch die anwendbare Gesetzgebung, vor allem die Umweltgesetze eingeschränkt.

\subsection{Demarkierung des indigenen Landes}

Die Demarkierung des indigenen Grund und Bodens ist eine Pflicht des Bundes, wie es der caput von Artikel 231 der Verfassung bestimmt, wonach neben der Demarkierung es auch die Pflicht des Bundes ist, es zu schützen und allen seinen Gütern Achtung zu verschaffen.

In Artikel 65 des Indianerstatuts (Gesetz Nr. 6.001/1973) wurde eine Frist von fünf Jahren zur Demarkierung des indigenen Landes festgelegt, die nicht eingehalten wurde. Daher wurde in der Bundesverfassung von 1988 eine neue Frist von ebenfalls fünf Jahren festgesetzt, die ebensowenig eingehalten wurde (Artikel 67 des Aktes der vorübergehenden Verfassungsbestimmungen). In jedem Fall beeinträchtigt die Nichteinhaltung dieser Fristen nicht die Pflicht, die Demarkierung abzuschließen, da es sich um Fristen ohne Verwirkungscharakter handelt, deren Ziel es nur war, die ungefähre Dauer der Durchführung der Arbeiten anzugeben.

Das Verfahren zur Identifizierung, Abgrenzung, dinglichen Demarkierung, Bestätigung und Registrierung des indigenen Landes ist in Dekret Nr. 1.775 vom 08. Januar 1996 geregelt. Die Zuständigkeit für die Initative und Anleitung des Verfahrens wurde dem Bundesorgan zur Unterstützung für die Indianer erteilt (Artikel 1 des Dekrets Nr. 1.775/1996), das derzeit die Nationale Indianerstiftung (FUNAI) ist.

Der erste Schritt dieses Verfahrens ist die Benennung einer Expertengruppe seitens der Nationalen Indianerstiftung, die vorzugsweise aus Beamten aus deren Mitarbeiterstab besteht und von einem Anthropologen geleitet wird, um die notwendigen ethno-historischen, soziologischen, juristischen, kartographischen und Umweltstudien durchzuführen sowie für die topographische Erhebung des Landes (Artikel 2, §1 des Dekrets Nr. 1.775/1996). Falls eine topographische Erhebung notwendig ist, kann diese von Bundesund bundesstaatlicher Behörde gemeinsam durchgeführt werden, und es ist auch möglich, die Mitarbeit der wissenschaftlichen Gemeinde und anderer staatlicher Organe zu erbeten (Artikel 2, § 2 und $\S 4$ des Dekrets Nr. 1.775/1996). Die Beteiligung der betroffenen indigenen Gemeinde ist in allen Phasen des Demarkierungsprozesses zugesichert (Artikel 2, § 3 des Dekrets Nr. 1.775/1996). Nach Abschluss der Arbeit zur Identifizierung und Abgrenzung der zu demarkierenden Fläche muss die Fachgruppe dem Bundesorgan zur Unterstützung für die Indianer einen detaillierten Bericht vorlegen, das seinerseits, wenn es ihn genehmigt, in einer Frist von fünfzehn Tagen nach dem Empfangsdatum die Zusammenfassung im Amtlichen Mitteilungsblatt der Union und im Amtlichen Mitteilungsblatt der föderativen Einheit veröffentlichen lässt. Der Veröffentlichtung muss eine Beschreibung und die Karte der Fläche beiliegen. Ebenso muss sie am Sitz der Gemeinde verbreitet werden, in dem sich die Fläche befindet (Artikel 2, § 7 des Dekrets Nr. 1.775/1996).

Bis neunzig Tage nach Veröffentlichung der Zusammenfassung können die Interessierten und die Regierung der Bundesstaaten und Gemeinden, in denen sich die identifizierte 
und abgegrenzte Fläche befindet, eine eventuelle Entschädigung beanspruchen oder mögliche Fehler im Ganzen oder in Teilen des Berichts aufzeigen (Artikel 2, § 8 des Dekrets Nr. 1.775/1996). Dazu müssen sie dem Bundesorgan zur Unterstützung für die Indianer die Gründe und die entsprechenden Beweise vorlegen (Herrschaftstitel, Sachverständigengutachten, Gutachten, Zeugenerklärungen, Photos, Karten, etc.). Diese Norm hat die meisten Streitigkeiten verursacht, da sie oft von nicht-indigenen Besetzern verwendet wird, um den Demarkierungsprozess zu Unrecht zu verschleppen. In jedem Fall werden diese Beschwerden von einer Spezialistengruppe des Bundesorgans zur Unterstützung für die Indianer geprüft, eine Stellungnahme verfasst und diese zusammen mit dem ganzen Verfahrensvorgang binnen sechzig Tagen nach der Veröffentlichung der Zusammenfassung des Berichts des Demarkierungsverfahrens an den Justizminister schickt (Artikel 2, § 9 des Dekrets Nr. 1.775/1996).

Nach Erhalt der Unterlagen hat der Justizminister nach Artikel 2, § 10 dreißig Tage, um sich zu äußern. Er kann a) per Portaria (Verordnung) die indigene Fläche erklären, die demarkiert werden wird; b) bestimmen, dass die notwendigen Maßnahmen getroffen werden, um mögliche Fehler oder Mängel des Prozesses zu beheben, die in einer Frist von neunzig Tagen zu erfüllen sind, oder c) den Bericht des Bundesorgans zur Unterstützung für die Indianer per begründeter Entscheidung verwerfen, mit der Begründung, der Vorgang falle nicht unter Artikel 231, § 1 der Verfassung fällt. Nach Prüfung des Berichts wird die physische Demarkierung des indigenen Lands durchgeführt, wobei das Verfahren per Dekret vom Präsidenten der Republik zu bestätigen ist (Artikel 5 des Dekrets Nr. 1.775/1996). Später wird die demarkierte Fläche beim Immobilienregister desjenigen Gerichtsbezirks registriert, in dem sie sich befindet, wobei sie auch beim Sekretariat für das Bundesvermögen des Finanzministeriums zu registrieren ist, was vom Bundesorgan zur Unterstützung für die Indianer in den dreißig auf die Veröffentlichung des Bestätigungsdekrets folgenden Tagen durchzuführen ist (Artikel 6 des Dekrets Nr. 1.775/1996). Andererseits wird in dem Fall, dass die Anwesenheit von nicht-indigenen Besetzern auf dem demarkierten Land festgestellt wird, die von der Nationalen Indianerstiftung geschaffene Ständige Untersuchungskommission die Bezahlung der auf dem indigenen Land realisierten Wertsteigerungen bestimmt, insofern es sich um Besetzer guten Glaubens handelt. Dabei ist die Haushaltsplanung des Bundes zu achten.

Die Demarkierung des Landes ist für eine indigene Gemeinschaft so grundlegend, dass die Indigenen im Fall des Versäumnisses den Bund gerichtlich belangen und die Erfüllung dieser verfassungsmäßig festgelegten Pflicht einfordern können (Artikel 231, caput). Dazu müssen die Voraussetzungen erfüllt sein, die für jede Gerichtsklage erforderlich sind: a) rechtliche Möglichkeit des Antrags (Artikel 295, einziger Paragraph, Abschnitt III der Zivilprozessordnung - Gesetz Nr. 5.869/1973); b) Legitimität für die Klage (Artikel 295, Abschnitt II der Zivilprozessordnung - Gesetz Nr. 5.869/1973); und c) prozessuales Interesse (Artikel 295, Abschnitt III der Zivilprozessordnung - Gesetz Nr. 5.869/1973). Außerdem müssen die Prozessvoraussetzungen in Bezug auf die Parteien vorhanden sein: a) Fähigkeit, Partei zu sein; b) Fähigkeit, vor Gericht zu stehen; c) Fähigkeit, bei Gericht zu 
fordern $^{11}$. In Bezug auf die letzte Voraussetzung ist die Verfügung in Artikel 232 der Verfassung zu berücksichtigen, wo die Beteiligung der Staatsanwaltschaft bei allen Akten von Gerichtsprozessen festgelegt wird, die Rechte von Indigenen involvieren. Schließlich ist in Bezug auf die Rechtsnatur des Verwaltungsakts der Demarkierung von indigenem Land hervorzuheben: Der Großteil der brasilianischen Rechtslehre verteidigt, dass es sich aufgrund der Festlegung im caput von Artikel 231 um einen Akt deklaratorischer Natur handelt, wo steht, dass den Indigenen die originalen Rechte auf das Land anerkannt werden, das sie traditionell in Besitz haben. Im gleichen Sinn lehrt Leitão: „Die Demarkierung indigenen Lands, Ergebnis der Anerkennung durch den Staat, ist also ein rein deklaratorischer Akt, dessen Ziel es einfach ist, die reale Ausdehnung des Besitzes zu präzisieren, um die volle Wirksamkeit der Verfassungsbestimmung zu sichern, die dem Staat die Pflicht auferlegt, es zu schützen"12.

Der Oberste Bundesgerichtshof - Supremo Tribunal Federal - hat sich zugunsten des deklaratorischen Charakters der Demarkierung des indigenen Landes in seiner Entscheidung zur Raposa Serra do Sol (PET 3.388-4) geäußert ${ }^{13}$ :

„,... Zusammenfassung: Popularklage. Demarkierung des Indigenen Lands Raposa Serra Do Sol. ...

3.3. Die administrative, vom Präsidenten der Republik bestätigte Demarkierung ist ein „Staatsakt, der der Annahme juris tantum von Legitimität und Wahrhaftigkeit dient" (RE 183.188, aus der Berichterstattung des Ministers Celso de Mello), außerdem ist er deklaratorischer Natur und selbst-vollstreckbar [...].

Und weiter in Dekret Nr. 1.775/1996 (Artikel 2, § 10, "I") gibt es einen direkten Bezug auf den deklaratorischen Charakter des Verfahrens, da dort ausdrücklich festgelegt wird, dass das Bundesorgan zur Unterstützung für die Indianer per Verordnung die Grenzen des indigenen Landes erklären und ihre Demarkierung bestimmen wird.

\subsection{Anwesenheit Dritter auf dem indigenen Land}

Nach Artikel 231, § 6 werden alle Akte, die die Besetzung, Eigentum- oder Besitznahme von indigenem Land zum Gegenstand haben, für nichtig und erloschen erklärt, wobei gutgläubigen Dritten nur das Recht eingeräumt wird, im Rahmen des Gesetzes eine Entschädigung für durchgeführte Verbesserungen zu empfangen. Das Gesetz, das diese Art und das Verfahren zur Bezahlung der Verbesserungen regelt, ist jedoch noch nicht verkündet wor-

Leandro Silva Raimundo, Dos pressupostos processuais e das condições da ação no processo civil. in: http://jus2.uol. com.br/ doutrina/texto.asp?id=5493\&p=2. Zugriff am: 16. Nov. 2009.

12

Raimundo Sérgio Barros Leitão, Natureza jurídica do ato administrativo de reconhecimento da terra indígena - a declaração em juízo, in: Santilli, Juliana (Coord.), Os direitos indígenas e a constituição, Porto Alegre, 1993, S. 67. Eigene Übersetzung.

13 Supremo Tribunal Federal. PET 3388/ RR Roraima. in: .http://www.stf.jus.br/portal/ jurisprudencia/listarJurisprudencia.asp?s1=\%283388.NUME.\%20OU\%203388.ACMS.\%29\&bas e=baseAcordaos. Zugriff am: 15. Okt. 2009. Eigene Übersetzung 
den, weshalb subsidiär Artikel 1.219 des Zivilgesetzbuchs (Gesetz Nr. 10.406/2002) angewandt wird, wo den gutgläubigen Besetzern folgende Rechte anerkannt werden: a) Entschädigung für die realisierten notwendigen und nützlichen Verbesserungen; b) Zurückhaltung der Verbesserungen, solange diese nicht entschädigt sind; und c) Entnahme von Luxusverbesserungen (zum Luxus oder zur Erholung), wenn dies der Immobilie keinen Schaden zufügt. Die Gutgläubigkeit des Besetzers bezieht sich auf die Unkenntnis, dass er indigenes Land besetzt. Bezüglich des Werts der Verbesserungen wird ein Gutachter diesen auf Grundlage technischer Kriterien bestimmen, wobei er dem tatsächlichen Wert so nah wie möglich zu sein hat. Schließlich wird der Bund für die Bezahlung der Verbesserungen verantwortlich sein.

\section{Entscheidung des Supremo Tribunal Federal über den Fall Raposa Serra Do Sol}

Für eine indigene Gemeinde ist das Land nicht nur die wirtschaftliche Überlebensquelle, sondern bildet die Referenzquelle ihrer Existenz selbst. So hat sich der Interamerikanische Menschenrechtsgerichtshof im Fall der Gemeinschaft Mayagna (Sumo) Awas Tingni gegen Nicaragua am 31. August 2001 geäußert $^{14}$ :

„149. [...] Die Indigenen haben aufgrund der Tatsache ihrer eigenen Existenz das Recht, frei auf ihrem eigenen Territorium zu leben; die enge Beziehung, die die Indigenen zu ihrem Land unterhalten muss als fundamentale Basis ihrer Kulturen, ihres spirituellen Lebens, ihrer Unversehrtheit und ihres wirtschaftlichen Überlebens anerkannt und verstanen werden. Für die indigenen Gemeinschaften ist die Beziehung zum Land nicht eine reine Frage von Besitz und Produktion, sondern ein materielles und spirituelles Element, in dessen vollen Genuss sie kommen müssen, auch um ihr kulturelles Legat zu bewahren und es an zukünftige Generationen weiterzugeben".

Gleichwohl unterliegt das Recht der Indigenen auf Land so, wie es mit jedem anderen Grundrecht geschieht, einigen Verfassungsbeschränkungen, die wir im Folgenden auf Grundlage des Urteils des Obersten Bundesgerichtshofs im Fall der Demarkierung des indigenen Landes Raposa Serra do Sol analysieren werden. In Brasilien erfüllt der Oberste Bundesgerichtshof im Einklang mit dem angewandten Modell zur Verfassungskontrolle neben der der Revisionsinstanz die Aufgabe des Verfassungshüters. Dieses Gericht kontrolliert eine führende und interventionistische Verfassung. Wie bedeutsam die hier aufgezeigten Unstimmigkeiten auch sein mögen, so erscheint die Reflexion angebracht, dass die Gewalt des brasilianischen Staats über sein Territorium und seine Verantwortung für den Schutz von Kultur und Räumen der indigenen Bevölkerungen wesentliche Themen für den konstitutionellen Dirigismus sind. Genau aus diesem Grund erscheint die Entscheidung im

Corte Interamericana de Derechos Humanos, Sentencia del 31 de agosto de 2001, Serie C - n 79, in: http://www.corteidh.or.cr/pais.cfm?id_Pais=15. Zugriff am: 15. Okt. 2009. Eigene Übersetzung. Einzelheiten schon bei Margret Carstens, Indigene kollektive Land- und Ressourcenrechte in der "Awas Tingni"-Entscheidung des Interamerikanischen Gerichtshofs für Menschenrechte, VRÜ 37 (2004), S. 236 ff. 
Allgemeinen zutreffend, da sie diesen führenden Charakter des brasilianischen Staates (und auf diese Weise in einer demokratischen Verfassung vorgeschriebenen) bestätigt, was, so wiederholen wir, die Entscheidung nicht von den Kritiken befreit. Der Kern des Schutzes der indigenen Bevölkerungen scheint beachtet zu sein, wobei diese Entscheidung immerhin die erste ist, die auf die Bedeutung der die indigenen Bevölkerung betreffenden Verfassungsnormen und deren Zusammenleben in einer sich als multikulturell begreifenden Gesellschaft ausführlich eingeht. Die Entscheidung des Supremo Tribunal Federal über die Demarkierung des indigenen Landes Raposa Serra do Sol (PET 3.388-4 Roraima ${ }^{15}$ ) wurde am 19. März 2009 gefällt und bildet trotz einiger Probleme, die im Folgenden erörtert werden, einen Meilenstein auf dem Weg zum umfassenden Schutz der indigenen Rechte in Brasilien.

Raposa Serra do Sol ist ein Reservat in den Gemeinden Normandía, Pacaraíma und Uiramutã im Bundesstaat Roraima mitten im brasilianischen Amazonasgebiet. Seine Fläche beträgt 1.747.464 Hektar und wird seit mehr als 150 Jahren von den Ethnien Macuxi, Patamona, Wapixana, Taurepang und Ingarikó bewohnt, die untereinander stark interagieren und deren Sprachen sich von einem gemeinsamen Stamm ableiten. Auf dem selben Land lebten ${ }^{16}$ auch Viehzüchter und Bauern, vor allem Reisbauern, die durch eine vom Nationalen Institut für Kolonisierung und Landreform (INCRA) durchgeführte Politik zur Kolonisierung von Amazonas unterstützt wurden, das ihnen in den letzten Jahrzehnten Eigentumstitel für dieses Landstücke gewährt hatte. Die Anwesenheit dieser beiden Menschengruppen mit gegensätzlichen Interessen in der Raposa Serra do Sol war in den letzten Jahren Ausgangspunkt verschiedener Gerichtsklagen (Popularklagen, Besitzklagen, öffentliche zivilrechtliche Klagen, etc.) in der Bundesgerichtsbarkeit (die bei Streitigkeiten über indigene Rechte gemäß Abschnitt XI von Artikel 109 der Verfassung zuständig ist), die vom Obersten Bundesgerichtshof zentralisiert worden waren (der die Funktionen des höchsten Organs der brasilianischen Gerichtsbarkeit und des Verfassungsgerichts nach caput von Artikel 102 der Verfassung vereint).

Unter diesen Klagen sticht die Popularklage PET 3.388-RR hervor, in der der Oberste Bundesgerichtshof zugunsten der durchgängigen Demarkierung des Indigenen Reservats Raposa Serra do Sol entschied, was einen Meilenstein im Umsetzungsprozess indigener Rechte darstellt, da es sich um die erste durchgängige Demarkierung eines indigenen Lands von großen Proportionen handelt. Ungeachtet dieser Errungenschaft wurden im selben Urteil 19 Bedingungen für die Ausübung dieses Rechts auf Land festgelegt, von denen einige aufgrund ihrer Unvereinbarkeit mit der Bundesverfassung, dem Übereinkommen 169

Supremo Tribunal Federal. PET 3388/ RR Roraima. Verfügbar unter: http:/www.stf.jus.br/portal/ jurisprudencia/listarJurisprudencia.asp?s1=\%283388.NUME.\%20OU\%203388.ACMS.\%29\&bas 16 e=baseAcordaos. Zugriff am: 15. Okt. 2009.

Mit dem Urteil sind sie verpflichtet, das indigene Land zu verlassen. 
der Internationalen Arbeitsorganisation - ILO (von Brasilien ratifiziert ${ }^{17}$ ) und mit Gesetzen wie dem Indianerstatut (Gesetz Nr. 6.001/1973) wirklich kontrovers sind, wie im Folgenden dargelegt werden wird.

1.- Das Recht der Indigenen auf Nutznießung der vorhandenen Reichtümer des Bodens, der Flüsse und der Seen auf ihrem Land kann immer dann relativiert werden, wenn es das relevante öffentliche Interesse des Bundes erfordert, wie in einem Zusatzgesetz festgelegt ist. Diese Bedingung wiederholt das, was in $\S 6$ von Artikel 231 der Verfassung festgelegt ist. Trotzdem ist es wichtig zu bemerken, dass neben dem relevanten öffentlichen Interesse des Bundes und der Voraussicht eines Zusatzgesetzes ${ }^{18}$, das den Beschränkungsprozess regelt, es auch notwendig ist, die indigenen Gemeinschaften über diese Maßnahme zu befragen, wie in Artikel 6 des Übereinkommens 169 der Internationalen Arbeitsorganisation bestimmt ${ }^{19}$,

„1. Bei der Durchführung der Bestimmungen dieses Übereinkommens haben die Regierungen

a) die betreffenden Völker durch geeignete Verfahren und insbesondere durch ihre repräsentativen Einrichtungen zu konsultieren, wann immer gesetzgeberische oder administrative Maßnahmen, die sie unmittelbar berühren können, erwogen werden;

b) Mittel zu schaffen, durch die diese Völker sich im mindestens gleichen Umfang wie andere Teile der Bevölkerung ungehindert auf allen Entscheidungsebenen an auf dem Wahlprinzip beruhenden Einrichtungen sowie an Verwaltungs- und sonstigen Organen beteiligen können, die für sie betreffende Maßnahmen und Programme verantwortlich sind;

c) Mittel zu schaffen, die es diesen Völkern ermöglichen, ihre eigenen Einrichtungen und Initiativen voll zu entfalten, und in geeigneten Fällen die für diesen Zweck erforderlichen Ressourcen bereitzustellen.

2. Die in Anwendung dieses Übereinkommens vorgenommenen Konsultationen sind in gutem Glauben und in einer den Umständen entsprechenden Form mit dem Ziel durchzuführen, Einverständnis oder Zustimmung bezüglich der vorgeschlagenen Maßnahmen zu erreichen“.

2.- Das Recht der Indigenen auf Nutznießung ihres Landes umfasst weder die Nutzung der Wasserressourcen noch der Energiepotentiale, die von der Genehmigung des Nationalkongresses abhängig sind. Diese Beschränkung widerspricht direkt der Verfassung, denn die Notwendigkeit einer Genehmigung vom Nationalkongress der Wasser- und Energieressourcen auf indigenem Land ist nur für Dritte anwendbar, wie $§ 3$ von Artikel 231 festlegt, wo auch bestimmt ist, dass der Nationalkongress vorher die betroffenen indigenen Gemeinschaften konsultieren sowie ihnen die wirtschaftliche Partizipation an den Einnahmen aus dieser Ausbeutung zusichern muss, Forderungen, die vom Obersten Bundesgerichtshof übergangen wurden.

Das Übereinkommen 169 der ILO wurde mit dem seit 2003 geltenden Legislativen Dekret Nr. 143 ratifiziert.

18

Ein Zusatzgesetz ist eine Art Normative, bei die Verfassung selbst die Fälle bestimmt, in denen es zu verwenden wird, wobei es dadurch charakterisiert ist, dass die absolute Mehrheit zur Beschlussfassung verlangt ist (Artikel 69 der Verfassung).

19 Internationale Arbeitsorganisation, Übereinkommen Nr. 169. in: http://www.gfbv.de/inhaltsDok. php?id=443. Zugriff am: 28.Feb. 2011. 
3.- Das Recht der Indigenen auf Nutznießung ihres Landes schließt weder das Schürfen nach, noch die Ausbeutung von mineralischen Bodenschätzen ein, was stets von der Genehmigung des Nationalkongresses abhängig ist, wobei den Indigenen im Einklang mit den gesetzlichen Bestimmungen Partizipation an den Einnahmen aus der Ausbeutung zugesichert wird.

Diese dritte Bedingung steht im Einklang mit der Verfassungsnorm, da das exklusive Nutznießrecht über die Reichtümer des indigenen Lands nur in Bezug auf Boden, Flüsse und Seen garantiert worden ist (Artikel 213, § 2), wobei Minen und Quellen für Wasserenergie ein vom Boden verschiedenes Eigentum bilden, deren Ausbeutung dem Bund gehört, die durch Privatleute per Genehmigung oder Konzession durchgeführt werden kann (Artikel 176, § 1). Andererseits ist erneut zu betonen, dass nicht die Notwendigkeit vergessen werden darf, zuvor die Indigenen zu konsultieren, wie es im Übereinkommen 169 der ILO festgelegt ist.

4.- Das Recht der Indigenen auf Nutznießung ihres Landes schließt weder das nichtindustrielle Schürfen (garimpagem) noch das Goldwaschen (faiscação) ein, wobei falls notwendig die entsprechende Erlaubnis eingeholt werden muss.

Diese vierte Beschränkung ist zu vernachlässigen, da, auch wenn es sicher ist, dass die Erzvorkommen, einschließlich der unterirdischen, Güter des Bunds sind (Artikel 20, IX der Verfassung) und dass ihre Ausbeutung durch Privatpersonen nur nach vorheriger Genehmigung oder Konzession durchgeführt werden kann (Artikel 176, § 1 der Verfassung), es notwendig $\mathrm{zu}$ betrachten ist, dass das Indianerstatut (Artikel 44 des Gesetzes Nr. 6.001/1.973) den Indigenen die Ausübung von nicht-industriellem Schürfen (garimpagem), Goldwaschen (faiscação) und die Suche (cata) nach Mineralien auf ihrem Land genehmigt, und folglich das Gesetz zu gelten hat und nicht die Entscheidung des Obersten Bundesgerichtshofs, der nicht über die Kompetenz verfügt, ein Gesetz zu modifizieren oder außer Kraft zu setzen, es sei denn, er übt die Kontrolle der Verfassungskonformität aus, was nicht der Fall dieses Prozesses ist.

5.- Das Recht der Indigenen auf Nutznießung ihres Landes überlagert nicht das Interesse der Nationalen Verteidigungspolitik. Die Errichtung von militärischen Basen, Einheiten, Posten und anderen militärischen Interventionen, die strategische Ausweitung des Straßennetzes, die Ausbeutung von Energiealternativen strategischer Natur und der Schutz strategischer Ressourcen werden nach Ermessen der zuständigen Organe (Verteidigungsministerium und Nationaler Verteidungsrat) unabhängig von den betroffenen indigenen Gemeinschaften und der Nationalen Indianerstiftung umgesetzt.

Diese fünfte Auflage steht in offenem Widerspruch zur Verfassung und zum Übereinkommen 169 der ILO, denn die Okkupation, Eigentums-, Besitznahme und Ausbeutung der natürlichen Reichtümer von Boden, Flüssen und Seen indigenen Landes kann allein im Fall von Katastrophen, Epidemien beschränkt werden oder zeitweise zur Bewahrung der nationalen Hoheit, wobei die Evakuierung der Indigenen von ihrem Land veranlasst werden kann, aber ihnen die sofortige Rückkehr garantiert wird, sobald das Risiko endet. Die Verfassungsnorm ist bei der Bestimmung kategorisch, dass der Schutz der nationalen Souverä- 
nität nur in dringenden und vorübergehenden Fällen angerufen werden kann, um die indigenen Rechte auf Besitz und Ausbeutung ihres Landes zu beschränken (Artikel 231, § 5 der Verfassung). Folglich darf eine nationale Verteidigungspolitik, die permanenten und nicht ausnahmsweisen Charakter hat, nicht die den Indigenen verfassungsmäßig garantierten Rechte überlagern. Aber noch im Dezember 2008 wurde das Dekret Nr. 6.703 erlassen, das die Nationale Verteidigungsstrategie regelte, ohne die auf indigenes Land anwendbaren Beschränkungen zu erwähnen, die den außerordentlichen Charakter offenbart, die diese Art Beschränkung mit sich bringt.

Andererseits widerspricht diese fünfte Beschränkung auch Artikel 6 von Übereinkommen 169 der ILO, wonach die indigenen Gemeinschaften das Recht haben, bei allen sie betreffenden Entscheidungen konsultiert $\mathrm{zu}$ werden und $\mathrm{zu}$ partizipieren, wie bei der Analyse der ersten Bedingung oben bereits dargelegt.

6.- Das Handeln von Streitkräften und Bundespolizei in indigenem Land ist im Rahmen ihrer Befugnisse garantiert und erfolgt unabhängig von der Konsultation der betroffenen indigenen Gemeinschaften und der Nationalen Indianerstiftung.

Diese sechste Beschränkung enthält dieselben Probleme wie die vorherige, sie widerspricht also frontal der Verfassung von 1988 und dem Übereinkommen 169 der ILO.

7.- Die Nutznießung der Indigenen unterbindet nicht die Errichtung von öffentlichen Anlagen, Kommunikationsnetzen, Straßen und Transportwegen durch den Bund sowie anderer für öffentliche Dienstleistungen notwendige Bauten durch den Bund, vor allem in Gesundheit und Bildung.

Wie bei der Analyse der ersten Bedingung beobachtet wurde, muss jede Beschränkung des Nutznießungsrechts der Indigenen drei Anforderungen genügen: a) relevantes öffentliches Interesse (Artikel 231, §6 der Verfassung); b) ein verfassungsergänzendes Gesetz, das die Beschränkung regelt (Artikel 231, § 6 der Verfassung); und c) Konsultation und Beteiligung der Indigenen an der Entscheidung (Artikel 6 des Übereinkommens 169 der ILO).

Indem sie nicht die Beteiligung der Indigenen bei den vom Bund zu realisierenden Bauten vorsieht, stellt diese siebte Bedingung daher einen Affront des Übereinkommens 169 der ILO dar.

8.- Das Recht der Indigenen auf Nutznießung in Gebieten, die von Schutzeinheiten betroffen sind, unterliegt der unmittelbaren Verantwortung des Instituts Chico Mendes zum Schutz der Biodiversität;

Das Thema der Überlagerung von indigenen Gebieten und Schutzeinheiten ${ }^{20}$ ist in dem Maße eines der polemischsten betreffend indigenes Land, indem es häufige Konfliktquelle

Schutzeinheit ist der per Gesetz mit dem Ziel errichtete territoriale Raum, die darauf existierenden natürlichen Ressourcen und Biodiversität zu erhalten. Das Gesetz Nr. 9.985/2000 schuf eine Nationales System von Schutzeinheiten (SNUC) das die Schutzeinheiten in zwei Gruppen teilt: a) integraler Schutz: erlaubt allein den indirekten Nutzen ihrer natürlichen Ressourcen: b) nachhaltiger Nutzen: versucht den Schutz der Natur mit dem nachhaltigen Nutzen seiner Ressourcen in Einklang zu bringen. 
zwischen indigenen Interessen und der Notwendigkeit des Umweltschutzes ist. In jedem Fall muss die Lösung eventueller Probleme ausgehend vom Dialog zwischen den Umweltschutzorganen (Institut Chico Mendes zum Schutz der Biodiversität und Brasilianisches Institut für Umwelt und Eneuerbare Natürliche Ressourcen ${ }^{21}$ ) und denen zum Schutz der Indigenen (Nationale Indianerstiftung) und den Indigenen selbst (nach der Bestimmung in Artikel 6 des Überkommens 169 der ILO) gefunden werden.

Indem die achte Beschränkung dem Institut Chico Mendes zum Schutz der Biodiversität die Verantwortung über die Nutznießung auf indigenem Land in Schutzeinheiten mit Exklusivität zuerkennt, widerspricht sie klar der Bestimmung in anderen Gesetzen und dem Übereinkommen 169 der ILO.

9.- Das Institut Chico Mendes zum Schutz der Biodiversität verantwortet die Verwaltung des Schutzeinheitsbereichs auf indigenem Land mit der Beteiligung der indigenen Gemeinschaften, die unter Berücksichtigung ihrer Gebräuche, Traditionen und Sitten gehört werden müssen, wobei sie dazu auf die Beratung der Nationalen Indianerstiftung zählen können.

Diese neunte Bedingung überwindet die Mängel der vorherigen, indem sie die Beteiligung der Indigenen und der Nationalen Indianerstiftung mit dem Institut Chico Mendes zum Schutz der Biodiversität zur Verwaltung der Flächen erwägt, wo es eine Überlagerung von Schutzeinheit und indigenem Land gibt.

Der Umweltschutz ist zweifellos eine Materie von relevantem öffentlichen Interesse und daher kann er geltend gemacht werden, um das Recht auf ausschließliche Nutznießung der Indigenen einzuschränken. Nichtsdestotrotz müssen, wie bei der ersten Bedingung dargelegt, einige Voraussetzungen erfüllt werden, ohne dass der Oberste Bundesgerichtshof in dieser Art Prozess über die Zuständigkeit verfügt, diese zu ändern.

10.- Der Verkehr von nicht-indigenen Besuchern und Forschern im von der Schutzeinheit betroffenen Gebiet muss in den Uhrzeiten und zu den Bedingungen zugelassen werden, die vom Institut Chico Mendes festgesetzt werden.

Da es sich um indigenes Land handelt, ist es nicht möglich, die Beteiligung der Nationalen Indianerstiftung und der Indigenen selbst von allen Entscheidungen auszuschließen, die die Rechte letzterer berühren. Folglich ist diese Beschränkung auch außer acht zu lassen, da sie der Verfassung und dem Übereinkommen 169 der ILO entgegensteht.

11.- Eintritt, Verkehr und Aufenthalt von Nicht-Indigenen in indigenem Land ist unter Achtung der von der Nationalen Indianerstiftung festgelegten Bedingungen zuzulassen.

Das Institut Chico Mendes zum Schutz der Biodiversität (ICMBIO) und das Brasilianische Institut für Umwelt und Erneuerbare Natürliche Ressourcen (IBAMA) wurden zu den ausführenden Organen des Nationalen Systems von Schutzeinheiten bestimmt (Artikel 6, III des Gesetzes Nr. 9.985/2000). Eine ihrer Ayfgaben ist es, in einer Frist von 180 Tagen nach Inkrafttreten des Gesetzes eine Arbeitsgruppe zu errichten, um Richtlinien zur Regulierung eventueller Überlagerungen von indigenen Flächen und Schutzeinheiten vorzuschlagen (Artikel 57), eine Aufgabe, die bis jetzt noch nicht vollständig erfüllt worden ist. 
Diese Bedingung verletzt das Übereinkommen 169 der ILO, indem sie ignoriert, dass die Indigenen bei den sie betreffenden Entscheidungen gehört werden müssen.

12.- Eintritt, Verkehr und Aufenthalt von Nicht-Indigenen kann nicht Gegenstand der Erhebung irgendwelcher Tarife oder Beträge irgendwelcher Natur seitens der indigenen Gemeinschaften sein.

Die Verfassung von 1988 hat in Artikel 231, § 2 das ausschließliche Nutznießungsrecht der Indigenen für den auf ihrem Land existierenden Boden, die Flüsse und Seen garantiert. In diesem Sinn ist ihnen verfassungsmäßig das Recht garantiert, diese Reichtümer auszubeuten, was zum Beispiel einschließen kann, von Dritten Geld zu erheben, um in dieses Land zu touristischen Zwecken einzutreten, auf ihm zu verkehren oder sich in ihm aufzuhalten. Was diskutiert werden kann, ist in jedem Fall nicht, ob die Indigenen dieses Recht haben, sondern die Vernünftigkeit oder Angemessenheit des erhobenen Betrags, was nur von Fall zu Fall bestimmt werden kann und nicht in der Form einer allgemeinen Bedingung, wie es in dieser Entscheidung des Obersten Bundesgerichtshofs geschehen ist.

13.- Die Erhebung von Tarifen oder Beträgen irgendwelcher Natur kann nicht auf die Autostraßen, staatliche Ausrüstungen, Hochspannungsleitungen oder andere in den Dienst des Publikums gestellte Geräte oder Installationen anfallen oder im Tausch für den Gebrauch verlangt werden, selbst wenn sie ausdrücklich von der Bestätigung ausgeschlossen wurden oder nicht.

Die Indigenen haben das Nutznießungsrecht des auf dem von ihnen traditionell besetzten Land existierenden Bodens, der Flüsse und Seen, auch wenn es sich um Bundeseigentum handelt (Artikel 231, § 2). Dieses Recht sichert ihnen jedoch nicht das Nutznießungsrecht anderer öffentlicher Güter zu, wie die in dieser Bedingung genannten, auch wenn sie auf ihrem Land vorhanden sind oder nicht im Bestätigungsdekret der Demarkierung des indigenen Lands erwähnt wurden. So hat diese Beschränkung nicht anderes getan, als die Reichweite der indigenen Nutznießung klarzustellen, ohne gegen die Bundesverfassung zu verstoßen.

14.- Indigenes Land darf nicht Gegenstand einer Verpachtung, eines anderen Aktes oder eines Rechtsgeschäfts sein, das die volle Ausübung der Nutznießung oder den direkten Besitz durch die indigene Gemeinschaft einschränkt.

Diese vierzehnte Beschränkung hat ihre Grundlage in der Verfassung selbst, wo ausdrücklich die Nichtigkeit und das Erlöschen der Rechtswirkungen aller Akte festgelegt sind, die die Besetzung, Herrschaft und Besitz von indigenem Land zum Gegenstand haben sowie die Ausbeutung der natürlichen Reichtümer der auf diesem existierenden Böden, Flüssen und Seen (Artikel 231, § 6). Diese Form ist mit dem Ziel vorgesehen, das Recht der Indigenen auf die permanente Besetzung ihres Landes $(231, \S 1)$ sowie das ausschließliche Nutznießungsrecht der auf diesem existierenden Ressourcen zu bewahren (231, § 2). So hat diese Bedingung nichts neues hinzugefügt, sondern vielleicht dazu beigetragen, ein wenig mehr die begrifflichen Konturen des indigenen Besitzes klarzustellen. 
15.- Allen den indigenen Stämmen oder Gemeinschaften fremden Personen ist es untersagt, auf indigenem Land Jagd, Fischerei oder das Sammeln von Früchten zu praktizieren sowie landwirtschaftliche Aktivitäten auszuüben.

Artikel 231, § 2 der Verfassung legt das Recht der Indigenen auf ausschließliche Nutznießung auf ihrem Land fest. Daher steht diese Beschränkung im Einklang mit der Verfassungsnorm.

16.- Das von den indigenen Gemeinschaften besetzte und besessene Land, die ausschließliche Nutznießung der natürlichen Ressourcen und der darauf existierenden Einrichtungen (unter Beachtung der Verfügung der Artikel 49, XVI, und 231, § 3 der Verfassung) sowie das indigene Einkommen genießen Steuerimmunität (imunidade tributária), wobei das Erheben von irgendwelchen Steuern, Gebühren oder Beiträgen darauf unzulässig ist.

Diese Bedingung wiederholt die Festlegung in Artikel 60 des Indianerstatuts (Gesetz Nr. 6.001/1973), wo festgelegt wird, dass die Güter und Einkommen aus indigenem Vermögen vollständige Steuerentlastung genießen, was eine wichtige Maßnahme zur Förderung der indigenen Rechte darstellt. Nichtsdestotrotz ist die terminologische Ungenauigkeit zu bemerken, die das Urteil des Obersten Bundesgerichtshofs aufweist, indem es das Wort „,imunidade“, das in Brasilien nur verfassungsmäßig vorgesehen werden kann, an Stelle von Befreiung verwendet, die per Gesetz, wie in diesem Fall, gewährt werden kann.

17.- Die Erweiterung des schon demarkierten indigenen Landes ist verboten.

Diese ist vielleicht die Bedingung, die am meisten Kontroversen hervorgerufen hat, denn sie hat nicht nur keine Verfassungsnorm zur Grundlage, sondern widerspricht dem Geist der Verfassung selbst, wo versucht wurde, eine Politik zur Förderung der indigener Rechte zu bekräftigen. Außerdem hat sich die Gerichtsbarkeit schon bei mehr als einer Gelegenheit aufgrund von Fehlern und Ungenauigkeiten bei der Identifizierung und Abgrenzung von als indigen anzusehenem Land über Fälle zur Revision von Demarkationsprozessen geäußert. Folglich verletzt das Verbot der Ausweitung der schon demarkierten Fläche nicht nur die Verfassung, sondern offenbart auch die Unkenntnis der Realität ${ }^{22}$.

18.- Die Rechte der Indigenen auf ihr Land sind unverfügbar, unveräußerlich und unabdingbar.

Die Unverfügbarkeit, Unveräußerlichkeit und Unabdingbarkeit indigenen Landes sind in $\S 4$ von Artikel 231 der Verfassung vorgesehen, wie unter Punkt 1.3 dieser Arbeit, „Klassifizierung des indigenen Landes“, erklärt worden ist. Also findet diese achtzehnte Einschränkung ausdrücklichen Rückhalt in der Verfassung.

19.- Es ist die effektive Beteiligung aller Einheiten der Föderation bei allen Etappen des Demarkierungsprozesses zu sichern. staat Maranhão) dar. Ministério Público Federal, Terra indígena Bacurizinho terá que ser demarcada em 180 dias, in: http://pfdc.pgr.mpf.gov.br/clipping/julho-1/terra-indigena-bacurizinho-teraque-ser-demarcada-em-180-dias, Zugriff am: 15 Nov. 2009. 
Mit dieser Bedingung bestätigte der Oberste Bundesgerichtshof die föderative Struktur des brasilianischen Staates (in Artikel 60, § 4, I der Verfassung vorgesehene unveränderliche Klausel) und die Achtung der Autonomie jeder Bundeseinheit neu. In diesem Sinn hat jede Entscheidung, wie die Demarkierung indigenen Landes, die die Reduzierung von Rechten, Befugnissen oder Kompetenzen irgendeiner der Einheiten der Föderation (Bund, Mitgliedsstaaten, Bundesdistrikt und Kommunen) nach sich ziehen kann, auf deren direkte Mitwirkung zu zählen. Es ist jedoch, wie vom Obersten Bundesgerichtshof an anderer Stelle dieses Urteils bemerkt, die notwendige institutionelle Führung des Bundes in Bezug auf schon demarkiertes indigenes Land als eine Form anzuerkennen, die harmonische Ausführung und das friedliche Zusammenleben aller Menschengruppen zu garantieren, die die brasilianische Gesellschaft bilden.

All dem ist hinzuzufügen, dass trotz des polemischen Inhalts einiger dieser Bedingungen ihre Formulierung geholfen hat, dem Thema der Indigenen-Rechte wichtigen Raum in der aktuellen juristischen Agenda zu verschaffen. Dies trägt zur Diskussion um die Definition der begrifflichen Konturen indigener Rechte und ihrer effektiven Umsetzung bei.

\section{Schluss}

Die 19 Bedingungen, die das STF im Fall Raposa Serra do Sol aufgestellt hat, zeigen, was auf der Basis der Verfassung von 1988 schon erreicht wurde, markieren zugleich aber auch, was noch erstritten werden muss.

Das Wissen um die Indigenen und ihre Rechte ist weiterhin begrenzt. Was Grund und Boden für diese Menschen bedeuten, wird weiterhin häufig unterschätzt. Für sie ist das eigene Land Existenzbedingung und nicht nur eine wirtschaftliche Kategorie. Indigenes Land nicht zu schützen, kommt der Vernichtung der indigenen Kultur gleich. Das ist schlechthin nicht hinnehmbar in einem Staat wie Brasilien, der sich als demokratisch versteht und die Menschenwürde wie auch die Gleichheit aller Menschen als tragende Verfassungsprinzipien propagiert. 